\title{
Explanations of grouping in immediate ordered recall
}

\author{
ROBERT W. FRICK \\ University of Colorado, Boulder, Colorado
}

\begin{abstract}
This article is about grouping in immediate ordered recall. The following findings are reported: (1) grouping a presentation improves recall, even when steps are taken to prevent rehearsal; (2) grouping primarily improves recall of the items adjoining the grouping, creating primacy and recency within groups; and (3) this primacy and recency are found even when single, isolated errors in recall are considered. These results suggest that the effects of grouping cannot be fully explained by rehearsal, chunking, or the number of directions in which an item can be transposed. It is suggested instead that (1) the auditory short-term store contains an unparsed and uncategorized representation that must be parsed and categorized just prior to recall, in a process of recovery; (2) items adjoining the boundary of a presentation are more easily recovered; and (3) grouping creates a boundary within the presentation. To support this explanation, a final experiment demonstrates an interaction between type of stimuli and serial position, with grouping most improving recall of adjoining phonemes.
\end{abstract}

This article is concerned with the paradigm of immediate ordered recall, in which a set of items is recalled in order immediately following their presentation. When items are presented acoustically, or when a visual presentation is subvocally recoded (Conrad, 1964), immediate ordered recall tests an auditory short-term store (ASTS).

Grouping a presentation improves immediate ordered recall (Adams, 1915; Ryan, 1969; Starr, 1923). For example, recall can be improved by inserting pauses into a presentation so as to group the presentation rhythmically (Ryan, 1969). Three plausible sources of the improvement produced by grouping are rehearsal, chunking, and the limited directionality of transposition errors. There is no disputing the possibility that these sources might contribute to the improvement produced by grouping. The question is, do these sources fully account for this improvement? In the current study, evidence will be presented suggesting that they do not. Then, an explanation of grouping will be proposed that is based upon the idea that items in ASTS need to be identified just prior to recall. According to this explanation, items by boundaries, including the boundary formed by grouping, are easier to identify. Finally, an experiment will be reported, the results of which support this explanation.

\section{Rehearsal}

The most common method of grouping is to insert one or two pauses into a presentation so as to group it rhythmically. These pauses provide opportunities for rehearsal, and any such rehearsal likely would improve recall of the

This research was partially supported by NIMH Training Grant MH-14617-11 to the University of Colorado. I thank Heather Fowkes and Butch Stevenson for assistance with testing subjects, and Darla Broberg and anonymous reviewers for reading a previous version of this manuscript. Reprint requests may be sent to Robert Frick, Department of Psychology, SUNY, Stony Brook, NY 11794-2500. rehearsed items. Thus, a contribution by rehearsal to improved recall does not seem controversial.

Can rehearsal fully explain the improved recall produced by grouping? Wickelgren (1964) assumed, at least for the purpose of his investigation, that "whatever else a grouping method is, it is a method of rehearsal" (p. 414). Chi (1976) also suggested that the purpose of grouping is to facilitate rehearsal. These authors seem to be saying that rehearsal can account for the effects of grouping.

One theory explaining the limited capacity of ASTS apparently is committed to the claim that the effects of grouping are due to rehearsal. It has been suggested that capacity might be limited by the rate of decay from ASTS (Baddeley, 1986; Schweickert \& Boruff, 1986). According to this "trace-decay" theory of limited capacity, there is a limit to how long an item can be retained in ASTS, which is estimated to be between 1.5 and $2 \mathrm{sec}$ (Schweickert \& Boruff, 1986). If an item is not recalled or rehearsed within this time period, it decays. When items are rehearsed, the capacity of ASTS is determined by the speed of rehearsal; when rehearsal is prevented, capacity is determined by the speed of presenting and reporting the items.

At first, the improved recall produced by inserting a pause into the presentation might seem to be evidence against the trace-decay theory. The pause would increase the time between presentation and report; hence, it should impair recall. However, the theory is easily saved by assuming that the subject rehearses the just-presented items during the pause of the presentation, and that this rehearsal "refreshes" the representation of those items in ASTS. This assumption does not explain why rhythmic grouping improves recall, but at least it explains why rhythmic grouping does not impair recall. To explain the improved recall, one could assume that the rehearsal actually strengthens the representation rather than merely main- 
taining it. Alternatively, if items could be rehearsed faster than they were presented, the time between rehearsal and report in a grouped presentation might be less than the time between presentation and report in an ungrouped, unrehearsed presentation.

Experiment 1 represents an attempt to answer the question, does grouping improve recall if rehearsal between groups is prevented? In studies of free recall, a distinction is usually made between elaborative rehearsal and maintenance rehearsal (Craik \& Watkins, 1973). Elaborative rehearsal, in the form of chunking, will be considered in the next section, so for the moment, the concern is with maintenance rehearsal.

Maintenance rehearsal is simply a rote repetition of information. The current literature suggests that there may be two types of rote rehearsal of auditory information (e.g., Baddeley, 1986; Baddeley \& Lewis, 1981; Reisberg, Smith, Baxter, \& Sonenshine, in press). Using the architecture of working memory suggested by Baddeley (1981, 1986), Naveh-Benjamin and Jonides (1984) suggested that one type of rote rehearsal might be the repetition of items in ASTS and a second type might be the repetition of the items in the central executive. The first type of repetition seems to correspond to subvocalization, and the second type seems to correspond to conscious imagery.

Empirically, the key distinction between the two types of rote rehearsal seems to be that the first type of rehearsal (subvocalization) is prevented by articulatory suppression, whereas the second type of rehearsal (conscious imagery) is not. Articulatory suppression is the technique of having the subject speak a repetitive phrase during the performance of a task (Murray, 1967). When people engage in articulatory suppression, they can still be conscious of the sounds of words they are reading (Baddeley, 1981, p. 20) and they can still make judgments on the basis of the sounds of visually presented stimuli, such as judging whether two words rhyme (Baddeley \& Lewis, 1981; Besner, Davies, \& Daniels, 1981). Thus, articulatory suppression does not eliminate the second type of rehearsal.

However, in tests of immediate ordered recall, when subjects engage in articulatory suppression while items are being presented visually, a variety of dependent measures have shown no evidence of the use of ASTS (e.g., Baddeley, Thomson, \& Buchanan, 1975; Conrad, 1972; Frick, 1985; Richardson, Greaves, \& Smith, 1980). These findings suggest that articulatory suppression prevents subvocalization and, hence, prevents the first type of rehearsal, rote repetition of the items in ASTS (Baddeley, Lewis, \& Vallar, 1984).

Wickelgren and Chi presumably would be content if either type of rote rehearsal was responsible for the improved recall produced by grouping. The trace-decay theory, however, would be committed to the hypothesis that subvocalization is responsible for the improved recall produced by grouping, because subvocalization would be needed to refresh the representation in ASTS (Baddeley et al., 1984).

In Experiment 1, two different methods were used to prevent rehearsal. First, subjects were required to engage in whispered articulatory suppression during the presentation. The normal method of articulatory suppression is to have the subject talk aloud during the presentation. This speech not only prevents subvocalization, it also creates acoustic noise, which can also impair the use of ASTS (Salame \& Baddeley, 1982). The second effect is desirable when the purpose of the articulatory suppression is to prevent the use of ASTS. However, in Experiment 1, the subjects were supposed to use ASTS; the purpose of the articulatory suppression was only to prevent rehearsal. Therefore, the subjects in Experiment 1 whispered the articulatory suppression. The sound of the articulatory suppression is not necessary to prevent subvocalization (e.g., Glassman, 1972; Klapp, Greim, \& Marshburn, 1981; Sannomiya, 1985).

Articulatory suppression is a good technique for preventing subvocalization, because it creates little impairment on tasks that do not require auditory rehearsal or auditory recoding (e.g., Klapp et al., 1981; Klapp, Marshburn, \& Lester, 1983). Articulatory suppression also does not prevent the use of ASTS when, as in Experiment 1, the presentation is acoustic (Klapp et al., 1981; Murray, 1968; Peterson \& Johnson, 1971).

In Experiment 1, two methods of grouping were tested. The first method was rhythmic grouping, in which a pause was inserted into the presentation. A pause is the most frequently used method of grouping an auditory presentation. However, inserting a pause into a presentation is also the method of grouping that most facilitates rehearsal. To prevent rehearsal, in Experiment 1 spatial grouping was also tested, in which one group of items was presented from one location and the second group of items was presented from a second location.

Changing spatial location does not provide extra time for rehearsal, so any opportunity to rehearse a spatially grouped presentation would also be available during ungrouped presentations. Changing spatial location might suggest a grouping strategy to the subjects. However, all subjects in Experiment 1 were encouraged to subjectively group the ungrouped presentation, and they would have known about grouping from having been previously tested with grouped presentations. Finally, changing location might provide a signal to the subjects for when to rehearse. However, Ryan (1969) found that inserting tones into a presentation to demarcate groups of items did not improve recall. This finding suggests that the mere signal to rehearse or group a presentation does not improve recall.

Yntema, Wozencraft, and Klem (1964; cited in Huttenlocher \& Burke, 1976) reported that spatial grouping improved recall. However, that study is unpublished, so the presence or absence of a pause between the presentations from the two locations cannot be ascertained. Furthermore, it seems unlikely that subjects in their control group would have been instructed to group the presentation subjectively. Therefore, the effect of the spatial grouping might have been merely to suggest a grouping strategy to the subjects. Frick (1988b) also reported that spatial grouping could improve recall. However, grouping was studied in a somewhat unusual situation: the con- 
trol presentation was grouped 4-5 and the experimental condition was grouped 4-4-1, so grouping of only the last item was tested. Recall of the last item of a presentation has been hypothesized to be governed by retention in a store other than ASTS (Crowder \& Morton, 1969). Thus, the available evidence suggests that spatial grouping probably improves recall, but the evidence is not as convincing as one might like.

\section{EXPERIMENT 1}

\section{Method}

\section{Subjects}

Thirty-two volunteers from introductory psychology classes at the University of Washington participated to earn extra credit toward their course grade.

\section{Stimuli}

The digits one through nine were used. Each digit was digitized using an analog-to-digital converter, sampling the sound every $100 \mu \mathrm{sec}$ and using approximately 128 gradations. The duration of each digit ranged from 256 to $448 \mathrm{msec}$. To present each digit during the experiment, the digitized representation was reconverted to an analog representation and presented on one speaker of a small stereo. A delay was inserted between the presentations of each digit, so that the duration from the middle of one digit to the middle of the next was approximately $475 \mathrm{msec}$.

For the rhythmically grouped presentations, the pause between the fourth and fifth digit was increased by an additional $300 \mathrm{msec}$. For the spatially grouped presentations, the subject sat in front of two speakers placed approximately $1 \mathrm{~m}$ apart, separated by approximately $90^{\circ}$. For each subject, the first four items were presented from the same spatial location on every trial. This location (right or left) was counterbalanced across subjects, orthogonally to the order of testing grouped and ungrouped presentations. When the presentation was ungrouped, the last four items were presented from the same location as the first four items; when the presentation was grouped, the last four items were presented from a different spatial location.

For each trial, eight different digits were presented. For each subject and each trial, the digits were selected randomly.

\section{Design}

There were two practice trials, one for grouped and one for ungrouped presentations. Then there were four blocks of experimental trials, each containing seven trials. Two of these blocks tested grouped presentations and two tested ungrouped presentations. Testing of grouped and ungrouped presentations was alternated across practice trials and blocks, and the condition tested first was counterbalanced across subjects.

\section{Procedure}

The procedure of the experiment was explained to the subjects before beginning, including the fact that some presentations would be grouped. The subjects were encouraged to subjectively group all presentations. Furthermore, before being tested with the ungrouped presentations, the subjects were always tested at least once with a grouped presentation (the practice trial), and for $75 \%$ of the ungrouped presentations, the subjects had been previously tested with at least eight grouped trials.

On each trial, a message appeared on the computer screen instructing the subject to begin whispering the word "the." The subject began whispering "the" at an even tempo, with no time between repetitions to subvocalize the items in the presentation. Two seconds later, the eight digits were presented. Immediately following the presentation, the subject stopped whispering "the" and attempted to report the digits aloud in the correct order.

\section{Results and Discussion}

The dependent measure was the percentage of trials on which a subject correctly recalled the correct item in the correct serial position. First, consider the rhythmically grouped presentations. There was a robust effect of grouping, with the subjects recalling $80 \%$ of the digits from the grouped presentation and only $70 \%$ of the digits from the ungrouped presentation $[t(15)=3.65, p<.01]$. The serial position curves for each condition are presented in Figure 1.

Next, consider the spatially grouped presentations. Grouping again improved recall, with the subjects recalling $80 \%$ of the digits in the grouped presentation and $72 \%$ of the digits in the ungrouped presentation $[t(15)=4.84$, $p<.001]$. The serial position curves for these two conditions are shown in Figure 2.

These results suggest that the effects of grouping are not dependent upon rehearsal. Thus, the results are difficult to explain within the trace-decay theory of limited capacity. However, they do not rule out the possibility that rehearsal contributes to the improved recall produced by grouping; they suggest only that there is a substantial improvement produced by grouping that cannot be accounted for by rehearsal alone.

\section{CHUNKING}

The best-known theory of limited capacity is that ASTS can retain $7 \pm 2$ chunks (Miller, 1956), with a chunk being defined as a "familiar unit." According to this theory, recall can be improved through a process called chunking, in which a stimulus is recoded into another stimulus having fewer chunks. In Miller's example, a string of binary digits (e.g., 011) was transformed into

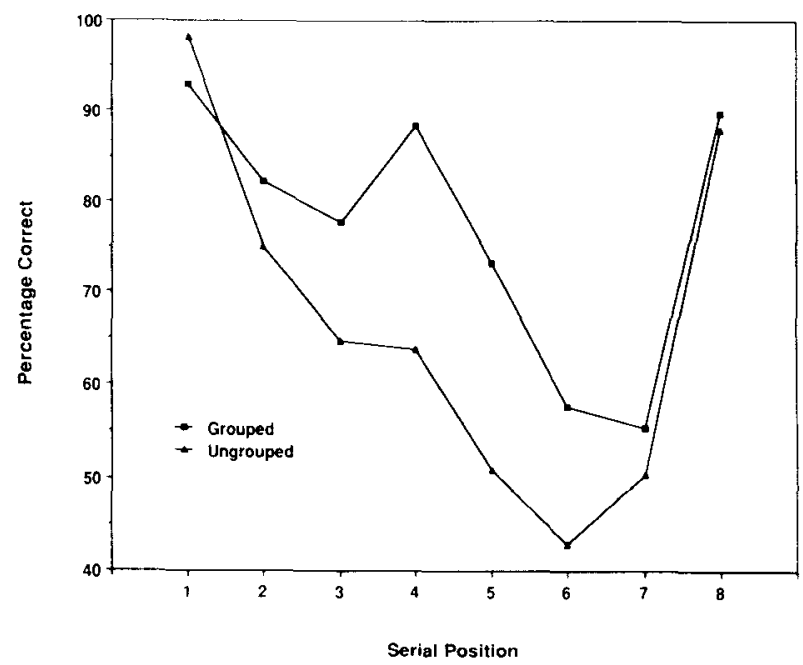

Figure 1. Serial position curves for the rhythmically grouped and ungrouped presentations in Experiment 1. 


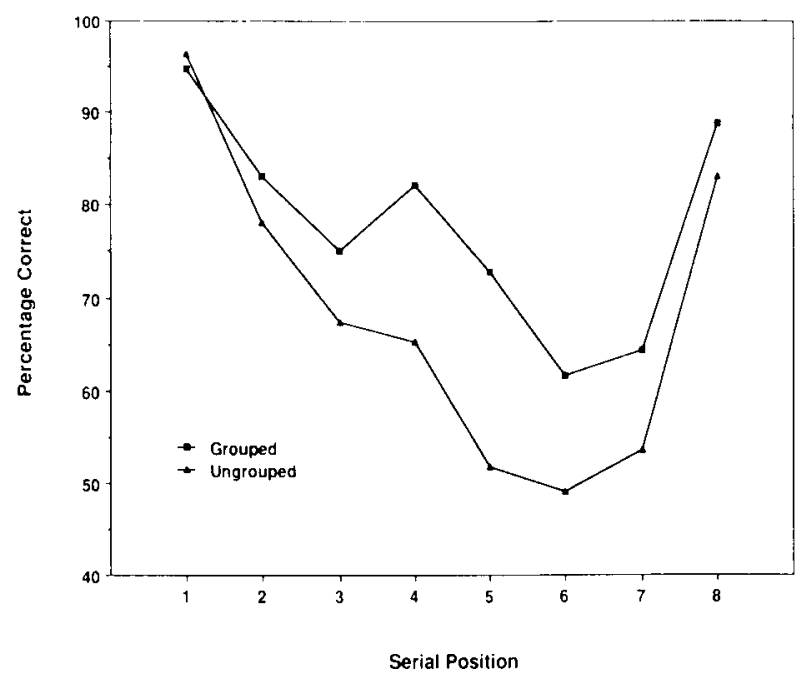

Figure 2. Serial position curves for the spatially grouped and ungrouped presentations in Experiment 1.

a decimal digit (e.g., 3). Another common example is that the three letters U-S-A can be recognized as an acronym for a North American country. The chunking explanation of grouping is that grouping facilitates chunking of the items in the presentation (Simon, 1974; Zhang \& Simon, 1985).

The evidence shows that organizing a presentation to reveal easily chunked groups of items will improve recall. For example, FBI-USA-PHD is easier to recall than either FBIUSAPHD or FB-IUS-AP-HD (Bower \& Springston, 1970). However, to show that chunking plays a prominent role in the improvement produced by grouping, two issues need to be resolved. The first issue is how easily subjects can chunk a quickly presented group of randomly selected digits. Clearly, 4-9-2 is not as easily chunked as is U-S-A. Any difficulty in chunking digits would be exacerbated by methods of grouping that do not include inserting a pause in the presentation. The second issue is whether physically grouping the presentation facilitates chunking any more than does simply instructing the subject to subjectively group the presentation.

One could argue that whenever a presentation is grouped, the items of a group are automatically chunked together. For example, in the presentation of 492-938571, 492 would automatically become one chunk. This concept of chunking is different than that in the previous examples. In the previous examples, the subject was aware of the chunking and could report the transformation of information. Furthermore, the resulting chunk was a familiar unit. In the above process, there is only a conjoining of symbols, and the result (e.g., 492) does not necessarily correspond to a familiar unit. It seems unfaithful to the original definition of chunking to call this process chunking; a better name might be conjoining. The argument is that by conjoining, 492 somehow becomes one chunk.

Because conjoining could occur automatically, there is no difficulty in explaining how it could occur with fast presentations, randomly selected digits, or a lack of processing time between the presentation of groups. One disadvantage of the conjoining explanation is that, unlike chunking, there is no independent evidence that conjoining improves recall. A second problem with the conjoining explanation is that grouping does not improve recall as much as would be predicted by conjoining, at least according to the following simple calculations. The lower limit to the capacity of ASTS is usually assumed to be around five chunks (e.g., Miller, 1956). Therefore, capacity for a presentation grouped in threes should be at least 15 items, and capacity for a presentation grouped in fours should be at least 20 items. The improvement produced by grouping seems to be in the range of about one or two items. This improvement is large and robust in the domain of immediate ordered recall, but it is not remotely as large as that predicted by conjoining.

As noted by Huttenlocher and Burke (1976), there is one piece of evidence against both chunking and conjoining. If inserting pauses into a presentation facilitates chunking or conjoining, then inserting pauses into a presentation should improve free recall. Instead, inserting pauses into a presentation does not improve free recall (Gianutsos, 1972).

Finally, consider the serial position curve for a grouped presentation. If a group of items is retained as a chunk, it should be forgotten as a chunk. Therefore, chunking would tend to flatten the serial position curves within groups.

There is another type of encoding, called magnitude encoding, in which the subject encodes the magnitude of the group of digits, as opposed to encoding the digits individually (Hinrichs \& Novick, 1982). This type of encoding might be considered a type of conjoining. Magnitude encoding produces a declining serial position curve, with good recall of the first digits and steadily declining recall of the later digits. Thus, magnitude encoding of groups would tend to produce a declining serial position curve within groups.

Several researchers have noted that there seems to be a primacy within groups (Huttenlocher \& Burke, 1976; Salame \& Baddeley, 1982) and recency within groups (Huttenlocher \& Burke, 1976; Ryan, 1969; Salame \& Baddeley, 1982). Within-group primacy and recency suggests that grouping primarily improves recall of the items adjacent to the grouping, and it is inconsistent with the hypotheses that the improvement produced by grouping is a result of chunking, conjoining, or magnitude encoding.

This presumed within-group primacy and recency apparently has never been verified statistically. In addition, the issue of primacy and recency within groups is not exactly the same as the question of which serial positions are improved most by grouping. The serial position curve for an ungrouped presentation tends to decline across serial positions (except for an upswing near the very end). Thus, if grouping improves all serial positions within a group equally, a slight primacy would still be expected within groups. 
To verify the primacy and recency within groups, and to determine which serial positions are improved most by grouping, the data from Experiment 1 were reanalyzed. The rehearsal explanation of grouping also predicts that grouping should equally improve the recall of all items of a group because all of the items are being rehearsed together. Therefore, the following analysis further tested the rehearsal explanation of grouping.

\section{Analysis 1}

In the following analysis, the data from the two methods of grouping used in Experiment 1 were combined. When the variable of method of grouping (rhythmic vs. spatial) was included in the following statistical tests, there were no main effects or interactions involving this variable, and the results of the statistical tests were essentially the same. Therefore, this variable was left out of the following statistical tests.

\section{Results}

The logical analysis to begin with is an ANOVA considering the variables of serial position and whether or not the presentation was grouped. There was a main effect of grouping $\left[F(1,31)=45.23, M S_{\mathrm{e}}=286, p<\right.$ $.001]$ such that grouping improved recall. This result simply reiterates the findings of Experiment 1 . There was also an interaction between grouping and serial position $\left[F(7,217)=10.48, M S_{\mathrm{e}}=111, p<.001\right]$, indicating that grouping improved the recall of some serial positions more than others. The interaction between grouping and serial position suggests a need to analyze each serial position individually.

The amount of increase produced by grouping at each serial position is presented in Table 1. The two largest increases occurred at Serial Positions 4 and 5, the two serial positions adjoining the grouping. These increases were both statistically significant, based upon a $t$ test comparing the grouped presentation to the ungrouped presentation for recall at that particular serial position $[t(31)=$ $7.22, p<.001$, for Serial Position 4, and $t(31)=7.02$, $p<.001$, for Serial Position 5].

The improvement in Serial Position 3 was also statistically significant $[t(31)=3.97, p<.001]$. However, it was not as large as the improvement in Serial Position 4. To verify this latter claim statistically, an ANOVA was performed with variables of grouping versus not grouping and Serial Position 3 versus Serial Position 4. The in- teraction was statistically significant $[F(1,31)=18.14$, $\left.M S_{\mathrm{e}}=49, p<.001\right]$, confirming that the grouping improved Serial Position 4 more than Serial Position 3. There also was a statistically significant improvement in Serial Position $6[t(31)=3.89, p<.001]$, but this improvement was not as large as the improvement in Serial Position $5\left[F(1,31)=5.99, M S_{\mathrm{e}}=86, p<.05\right]$. The improvement at Serial Position 7 was even smaller, though statistically significant $[t(31)=2.26, p<.05]$, and the improvements at Serial Positions 2 and 8 were small and statistically nonsignificant $[t(31)=2.01$, and $t(31)=1.50$, respectively]. Finally, at Serial Position 1, there was a statistically significant advantage for the ungrouped presentation $[t(31)=2.37, p<.05]$.

There is a clear pattern in these data: the largest improvements in recall were at the two serial positions adjoining the grouping, with a steady fall off in improvement for serial positions farther from the grouping.

In fact, it is possible to argue that the only direct effect of grouping is to improve recall of the adjoining items, and that the improvement in the other items is mediated by the improvement in the adjoining items. The argument is as follows. When Serial Position 5 was recalled, the probability of recalling Serial Position 6 was 76\% (across both experiments); when Serial Position 5 was not recalled, the probability of recalling Serial Position 6 was $15 \%$. Assuming these values are fixed, the $22 \%$ increase in Serial Position 5 produced by grouping would lead to a $13 \%$ increase in Serial Position 6. The actual increase in Serial Position 6 was $14 \%$. Similarly, the probability of recalling Serial Position 3 was $84 \%$ when Serial Position 4 was recalled and $29 \%$ when Serial Position 4 was not recalled. Therefore, the $21 \%$ increase in Serial Position 4 can account for a $12 \%$ increase in Serial Position 3, with the actual increase in Serial Position 3 being $10 \%$. Thus, the increased recall of Serial Positions 4 and 5 can, in theory, account for the increased recall of Serial Positions 3 and 6 .

There is no guarantee that the assumptions underlying the above mathematical calculations are correct. Therefore, the above argument does not demonstrate that the only direct effect of grouping is on the two adjoining serial positions. The argument shows only that the data are consistent with this hypothesis.

The same argument cannot be made for the relationship between Serial Positions 4 and 5. The probability of recalling Serial Position 5 was 79\% when Serial Position 4 was recalled and $41 \%$ when it was not. Therefore,

Table 1

Increase in Percentage of Recall Produced by a 4-4 Grouping Over an Ungrouped Presentation

\begin{tabular}{lrrrrrrrr}
\hline & \multicolumn{7}{c}{ Serial Position } \\
\cline { 2 - 8 } & 1 & 2 & 3 & 4 & 5 & 6 & 7 & 8 \\
\hline Experiment 1 & -4 & 6 & 10 & 21 & 22 & 14 & 8 & 4 \\
Frick (1989)-vocalized & -5 & 4 & 4 & 22 & 21 & 13 & 9 & 7 \\
Frick (1989)-subvocalized & -7 & -4 & -4 & 11 & 5 & -1 & -3 & 0 \\
\hline
\end{tabular}


the $21 \%$ improvement in Serial Position 4 can account for only an $8 \%$ improvement in Serial Position 5. Similarly, the probability of recalling Serial Position 4 was $92 \%$ when Serial Position 5 was recalled and $68 \%$ when it was not. Therefore, the $22 \%$ improvement in Serial Position 5 can account for only a $5 \%$ improvement in Serial Position 4. Thus, grouping must be having a direct effect upon the two serial positions adjoining the grouping.

The issue of primacy and recency within groups requires a slightly different statistical analysis, in which only the grouped presentation is considered. Serial Position 4 was recalled more often than Serial Position $3[t(31)=$ $4.10, p<.001$ ], demonstrating a within-group recency. Similarly, Serial Position 5 was recalled more often than Serial Position $6[t(31)=5.49, p<.001]$, demonstrating a within-group primacy.

\section{Discussion}

These results demonstrate that the grouping in Experiment 1 primarily improved recall of the items adjoining the grouping. The selective improvement in those serial positions leads to primacy and recency within groups. In fact, the results are not inconsistent with the possibility that the only direct effect of grouping is to improve recall of the two items adjoining the grouping. This pattern of effects on serial position cannot be explained by chunking, conjoining, magnitude encoding, or rehearsal. Thus, there must be some other source of the improved recall produced by grouping.

Experiment 1 was designed to prevent rehearsal, and the lack of a pause between groups of the spatially grouped presentation probably was not conducive to chunking. One could argue that if rehearsal had not been prevented and had chunking not been impaired, the serial position curves within groups might have been flatter. This argument does not challenge the conclusion that there is a source of grouping other than rehearsal and chunking. Nonetheless, one can ask the empirical question, would grouping have improved recall of primarily the adjacent serial positions if no attempt had been made to prevent rehearsal?

The observations by other authors of within-group primacy and recency suggests that these phenomena occur when rehearsal is not prevented. I coincidentally had performed another experiment (Frick, in press) that contained both an ungrouped presentation of eight items and a presentation grouped 4-4 by a pause. In that experiment, (1) nothing was done to elicit subjective grouping in the ungrouped presentation; (2) the total time of presentation was held constant, with the delay produced by the pause being compensated for by a slightly faster presentation of the items; and (3) the items were presented visually and the subjects either audibly vocalized each item as it was presented or subvocalized each item. The results of that experiment are included in Table 1. Those results suggest that even when no attempt is made to prevent rehearsal, grouping nonetheless primarily improves recall of the adjoining serial positions.

\section{BOUNDARY EFFECTS}

The effects of grouping upon the serial position curve suggest that there is something special about the boundaries of a presentation. For an ungrouped presentation, the boundaries are the start and the end of the presentation, and these items have an advantage in recall. Grouping apparently creates a boundary within the presentation, giving the two items adjoining the grouping an advantage in recall.

Harcum (1967) hypothesized that boundary items are remembered better because they are unique, and Murdock (1974) hypothesized that the better memory was a result of the boundary items being distinctive. These explanations are difficult to test because there is no method of measuring uniqueness or distinctiveness. In fact, it seems that the boundary items are no more unique or distinctive than the other items of the presentation, except that they are easier to recall.

Wickelgren $(1964,1967)$ suggested that there were three positional cues for recall: beginning, middle, and end. Postulating three cues explains why grouping in threes is the most effective pattern, but it does not explain the primacy and recency found within groups. Hypothesizing only two cues, beginning and end, would explain the within-group primacy and recency but would leave unexplained why grouping in threes is more effective than grouping in twos. In either case, there is no explanation of what these cues are or how they improve recall.

A more complex attempt to deal with the boundary effects is the positional cuing theory (Hendrikx, 1984; Sanders, 1975; Sanders \& Willemsen, 1978). According to this theory, the first item of each group receives a primacy tag and the last item of each group receives a recency tag. These "tags" provide access to recall of the items. For example, the first item of a presentation is recalled directly by its primacy tag, whereas the second item of the presentation is recalled by recalling the first item. The better recall of the boundary items follows from the fact that recall of the interior items is dependent upon recall of the boundary items.

The positional cuing theory provides little description of how the tags are formed or how they operate to provide access to the items. However, this theory does account for the fact that, for probed recall, boundary items are recalled faster than nonboundary items (Hendrikx, 1984; Sanders \& Willemsen, 1978). One criticism of the data supporting this theory is that, in probed recall, each item must be associated with a cue. It is possible to argue that the data reflect only how the items of the presentation are associated with the cues. For example, there may be direct access to the second item in ASTS, but there may be no method of associating the second item with the second cue.

These hypotheses all agree that there is something special about the boundaries of a presentation. The questions 
are, what is this special property (or properties), and how does it improve recall?

The limited directionality of transposition errors. A common error in immediate ordered recall is transposing two items, such that the order of report of the two items is reversed. The most likely transpositions are between two adjacent items. Transpositions between two items separated by another item (e.g., Serial Positions 1 and 3) are rarer, and transpositions between two items that are separated by two or more other items (e.g., Serial Positions 1 and 4) are even rarer still (Ryan, 1969).

Suppose the probability of a transposition was the same for any two adjacent serial positions. The first and last items of a presentation are adjacent to only one other item, so they can be transposed in only one direction, whereas the other items of the presentation can be transposed in two directions. Thus, the first and last items of a presentation would be only half as likely to be transposed as the other items in the presentation. This limited directionality of transposition errors predicts the existence of primacy and recency (Estes 1972; Lee \& Estes, 1977, 1981). To explain the within-group primacy and within-group recency, all that needs to be added is the observable fact that transpositions tend not to occur between items of different groups. Thus, the items adjoining the grouping can be transposed in only one direction, accounting for the boundary effects.

An important assumption of the above explanation is the underlying equivalence between serial positions: the probability of transposing any two adjacent serial positions is assumed to be equal. This assumption is not necessary for the mechanisms of limited directionality to operate. For example, one could assume that there was an underlying primacy and recency, which might then be augmented by the limited directionality of transposition errors. However, for the limited directionality of transposition errors to be a complete explanation of the boundary effects, there can be no underlying differences between the serial positions.

Analysis 2 tested the assumption of an underlying equivalence between serial positions. Consider trials in which the subject's only error is to transpose two adjacent items. If all serial positions were created equal, these isolated transpositions should be evenly distributed among the pairs of adjacent serial positions (except for the pair of serial positions spanning the two groups).

Consider also trials in which the subject's only error is to report one incorrect item in the place of one correct item. There are a variety of ways that isolated substitution errors might occur, including interference from previous trials (Lee \& Estes, 1981) and transposing parts of the item so that the item is not recognizable (Lee \& Estes, 1977). However, if there are no underlying differences between serial positions, the serial position curve of isolated substitution errors should also be flat.

To test these predictions, serial position curves were constructed from trials in which only an isolated transposition error or an isolated substitution error was made. These types of trials did not occur very often. Therefore, the data from Experiment 1 was supplemented with the data from the experiment mentioned in Analysis 1, which also grouped a presentation $4-4$.

\section{Analysis 2}

\section{Method}

Errors in recall were taken from Experiment 1 and another experiment reported elsewhere (Frick, in press). In that experiment, eight digits were presented one at a time on a computer screen at the rate of approximately one digit per $815 \mathrm{msec}$. The pause between the fourth and fifth digit was an additional $450 \mathrm{msec}$. On half of the trials, the subject spoke each item aloud as it was presented; on the other half of the trials, the subject said the digit subvocally. Errors were taken from both types of trials. Subjects spoke their answer immediately following the presentation.

Errors were collected from trials in which the only error was to make a single transposition of two adjacent items or a single substitution error. A total of 94 transpositions were collected from 50 subjects. The rhythmic grouping in Experiment 1 contributed 25 transpositions, the spatial grouping contributed 15 transpositions, and the outside experiment contributed 54 transpositions. A total of 99 substitution errors were collected, involving 45 subjects. These consisted of 19 errors from the rhythmic grouping in Experiment 1 , 13 errors from the spatial grouping, and 67 errors from the outside experiment

A sign test was used to compare errors at adjacent serial positions or adjacent pairs of serial positions, with the critical statistic being the serial position or pair of serial positions at which a subject had made fewer errors.

\section{Results}

Consider first the isolated transposition errors. The serial position curve is presented in Figure 3, with the abscissa representing pairs of adjacent serial positions. Serial Positions 2 and 3 were reversed more often than Serial Positions 1 and $2[+=12,-=1, p<.01]$ and more often than Serial Positions 3 and $4[+=14,-=3$, $p<.05]$. Similarly, Serial Positions 6 and 7 were reversed more often than Serial Positions 5 and $6[+=25$, $-=7, p<.01]$ and more often than Serial Positions 7 and $8[+=27,-=2, p<.001]$.

The serial position curve for isolated substitution errors is presented in Figure 4. There were more errors at



Figure 3. Serial position curve of single transposition errors. 


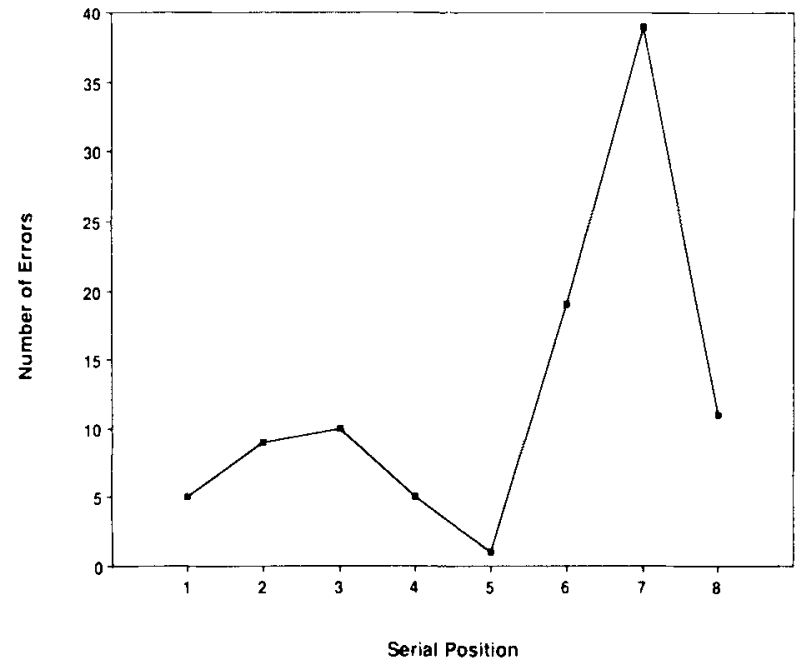

Figure 4. Serial position curve of single substitution errors.

Serial Positions 2 and 3 than at Serial Positions 1 and 4 , but the number of errors at these serial positions was small and the differences were not statistically significant. However, there were enough errors to make comparisons at Serial Positions 5 through 8. There were more errors at Serial Position 6 than at Serial Position $5[+=16$, $-=0, p<.001]$ and more errors at Serial Position 7 than at Serial Position $8[+=24,-=6, p<.01]$.

\section{Discussion}

The results of this analysis suggest that there are underlying differences between the serial positions: at the boundary of a presentation, transpositions and substitutions are less likely to occur. The limited directionality of transposition errors might augment these underlying differences between serial positions. However, the question is, what produces these underlying differences between serial positions?

Boundary effects in recovery. Elsewhere (Frick, 1988a), I have argued for the following theory concerning representation and limited capacity in ASTS. The representation in ASTS is precategorical, even to the extent of containing a representation more primitive than phonemes. The representation is also unparsed, in the sense that there are no divisions marking where one item ends and another starts.

However, the subject's report obviously is categorical, in that the subject attempts to report an instance of the item that was presented and does not mimic the pitch, loudness, or accent of the presentation. Therefore, the representation in ASTS must be parsed and categorized prior to recall, in a process called recovery. The representational medium in ASTS is assumed to be fixed and to be allocated proportionally to the items in the presentation. The capacity of ASTS, then, is the largest number of items that can be represented with sufficient fidelity to be recovered. Because so much is hypothesized to oc- cur in the process of recovery, this theory will be called the recovery theory.

The recovery theory provides the following explanation of the boundary effects. A logical property of any unparsed and uncategorized representation is that the items adjoining the boundaries are easier to identify. This property would apply to a string of written words if there were no spaces between words, and it applies to ASTS, assuming the representation in ASTS is precategorical and unparsed. Thus, knowing one edge of an item in an otherwise unparsed representation would facilitate identification of that item during recovery.

For example, suppose recovery started at the beginning of the representation in ASTS and the sound "ee" was found. The next logical step would be to add subsequent sounds to see if a word could be identified. Similarly, if "ee" was found at the end of the representation in ASTS, preceding sounds should be added. However, if "ee" was found in the middle of the representation, there would be three possibilities: adding preceding sounds, adding subsequent sounds, or adding both preceding or subsequent sounds.

Now consider what happens when "eet" is found in the start of the representation. This could be either a word (" "eat") or the start of a word (e.g. , "eating"). The next step is to analyze the subsequent sounds to see if they form a continuation of the first word or the start of a new word. Similarly, if "eet" is found at the end of the representation, preceding sounds should be analyzed. However, if "eet" is found in the middle of the representation, it might be a word ("eat"), the start of a word (e.g., "eating"), the end of a word (e.g., "defeat"), the middle of a word (e.g., "unbeatable"), or it could span word boundaries (e.g., "free time"). These possibilities are numerous, and there is no logical next step for resolving which possibility is correct.

Thus, there is no simple method of recovering items starting from the middle of the representation in ASTS, but recovery can potentially be started from any boundary of the presentation. This advantage in recovery for the items by the boundary provides a source of the boundary effects in immediate ordered recall. To what extent such a source is needed to explain first-item primacy and last-item recency is considered in Frick (1988a). More germane to the present topic, this theory accounts for the improved recall produced by grouping: grouping would create a boundary within the representation, allowing the items adjoining the grouping to be more easily identified in the process of recovery.

The recovery explanation of grouping is consistent with the findings presented previously in this article. First, rehearsal would not be necessary for grouping to improve recall. In fact, because the effects of grouping occur in the stage of recovery, the subject does not have to engage in any activity during the presentation for grouping to improve recall. This accounts for the fact that recall can be improved by methods of grouping other than in- 
serting a pause into the presentation. Second, grouping would be expected to primarily improve recall of the items adjoining the grouping. These boundary effects would $\propto-$ cur even for isolated transposition and substitution errors. Finally, the recovery explanation explains why the boundaries are the access points to the items in a representation. Thus, it is consistent with the finding that boundary items can be recalled more quickly than nonboundary items in tests of probed recall (Hendrikx, 1984).

Although the recovery explanation accounts for all of the effects presented above, it seems to do so in a mundane fashion. Therefore, an attempt was made to construct an unusual prediction from the recovery explanation. The recovery explanation predicts that the precise effect of grouping might depend upon the nature of the stimuli being tested. Consider the set of stimuli $<$ DATE, FATE, KATE, LATE, MATE, PATE, TATE, VATE, ZATE >. According to the recovery explanation, grouping a presentation of these items in the pattern 3-3 should mark the end of the third item and the start of the fourth item. Because the end of the third item is redundant and the start of the fourth item is critical, the grouping should benefit the fourth item more than the third. The opposite prediction would be made for the set of stimuli <TADE, TAFE, TAKE, TALE, TAME, TAPE, TATE, TAVE, TAZE >, in which the end of the item is critical and the start of the item is redundant. In this case, the grouping should benefit the third item more than the fourth.

In Experiment 2, recall of the preceding two sets of stimuli was tested. If the above prediction is correct, there should be an interaction between type of stimuli and Serial Positions 3 and 4.

There were several reasons for not comparing the grouped presentation to an ungrouped control condition. First, subjects in a control condition would probably group the presentation subjectively. Therefore, a control condition might show some of the same effects found in the grouped presentations. Second, subjects in a control condition might use a pattern of grouping other than 3-3. In that case, comparing the experimental condition to the control condition would have resulted in a comparison between two different patterns of grouping. Finally, an interaction between type of stimuli and Serial Positions 3 and 4 was sufficient to test the above hypothesis. This is an unusual prediction that apparently no other theory makes. Therefore, testing of an ungrouped presentation was not needed.

\section{EXPERIMENT 2}

\section{Method}

\section{Subjects and Design}

Thirty-two students from introductory psychology classes at the University of Colorado participated voluntarily to fulfill part of their course requirement.

\section{Stimuli}

Half of the stimuli were from the set <DATE, FATE, KATE, LATE, MATE, PATE, TATE, VATE, ZATE $>$ and the other half of the stimuli were from the set $\angle$ TADE, TAFE, TAKE, TALE, TAME, TAPE, TATE, TAVE, TAZE $>$. The former set will be called the -ATE stimuli and the latter set will be called the TA-E stimuli. The two sets contained the same phonemes and differed only in the location of the critical phoneme. The phonemes were selected to be as discriminable as possible.

For each trial, six different items were selected randomly from one of the sets. The items were presented one at a time in the center of a computer screen at the rate of 1 per $750 \mathrm{msec}$. An additional pause of approximately $365 \mathrm{msec}$ was inserted between the third and the fourth items, grouping the presentation rhythmically. There was no control over a subject's pitch and loudness, so other methods of grouping the presentation were probably also used. Of course, for the purposes of this experiment. it is sufficient to know that the presentation was grouped 3-3.

\section{Design}

The 32 experimental trials were divided into four blocks. Each block contained eight trials using items from one of the sets of stimuli. In addition, there were two practice trials, one for each set of stimuli. Testing of the two sets was alternated across practice trials and blocks of the experiment, and the set tested first was alternated across subjects.

\section{Procedure}

Before beginning the experiment, the subjects were told that this was a test of auditory short-term memory. They were told that they would be saying the items aloud in order to form an auditory image, which they should use in responding. Furthermore, they were instructed not to agonize over any particular choice, but instead just to report an answer quickly and not be too concerned about being correct. The purpose of these instructions was to induce the subjects to use auditory short-term memory. In most tests of immediate ordered recall, using auditory short-term memory is not only a good strategy, it is the only useful strategy. In the present experiment, using auditory short-term memory was not as effective as normal, because the items sounded alike. Furthermore, the items differed in only one letter, so other strategies involving remembering single letters were more effective than usual. The encouragement to use ASTS seemed to be effective.

Before beginning testing, the subjects were asked to read the stimuli twice, once when the items were presented simultaneously and once when the items were presented one at a time. Before each practice trial and each block of trials, the stimuli were read aloud to the subject. Each trial began with a message saying "READY" and another saying "BEGIN." Then the items were presented one at a time. The subject read each item aloud as it was presented. Immediately following the presentation, the subject attempted to report aloud all six items in the correct order, beginning with the first item. Guessing was encouraged, but the subject was allowed to say "blank" or make some other indication when he or she could not guess at a particular item. The subject had as long as needed to respond. The experimenter recorded the subject's response and then pressed a key to initiate the next trial. There were approximately $5 \mathrm{sec}$ between the end of the subject's response and the start of the next trial.

\section{Results}

The dependent measure was again the percentage of trials on which the subject recalled the correct item in the correct serial position. The serial position curves for the two types of stimuli are shown in Figure 5. The critical test involved a $2 \times 2$ ANOVA, with variables of type of stimuli (-ATE vs. TA-E) and serial position ( 3 vs. 4 ). There was a main effect of type of stimuli $[F(1,31)=$ 


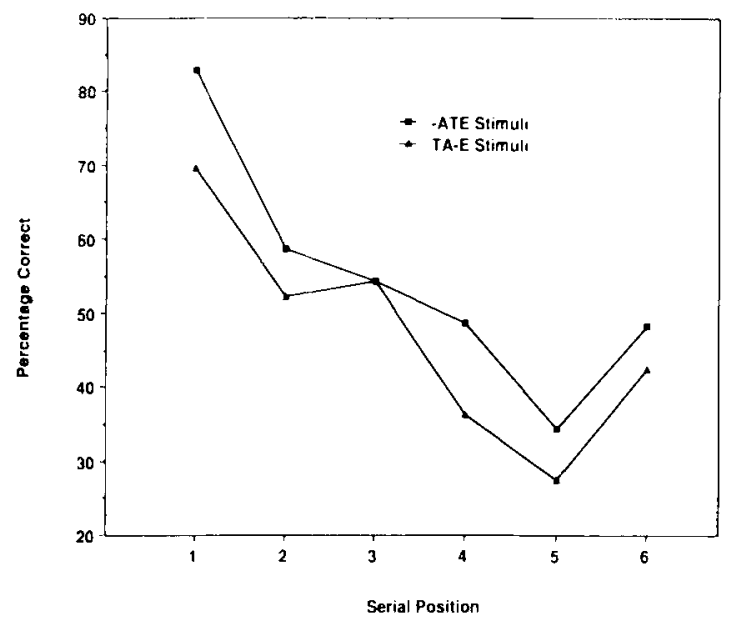

Figure 5. Serial position curves from Experiment 2.

7.26, $\left.M S_{\mathrm{e}}=167, p<.05\right]$ such that the -ATE stimuli were recalled more often than the TA-E stimuli. There was also a main effect of serial position $[F(1,31)=17.76$, $\left.M S_{\mathrm{e}}=254, p<.001\right]$ such that Serial Position 3 was recalled more often than Serial Position 4. Most importantly, there was an interaction between type of stimuli and serial position $\left[F(1,31)=8.45, M S_{\mathrm{c}}=144, p<\right.$ $.01]$. This interaction was in the direction predicted by the recovery explanation of grouping: the advantage of the -ATE stimuli was only at Serial Position 4; at Serial Position 3, recall of the two sets of stimuli was equal.

According to the recovery explanation, the same principle that applies to the items adjoining the grouping should also apply to the first and last items of the presentation: the -ATE stimuli should have an advantage for the first item, because the critical information is closer to the starting boundary, whereas the TA-E stimuli should have an advantage for the last item, because the critical information is closer to the ending boundary. For Serial Position 1, recall of the -ATE stimuli was better than recall of the TA-E stimuli $[t(31)=5.69, p<.001]$. Interestingly, this difference appeared even for recall of TATE, which appeared in both sets: when TATE was the first item presented, it was recalled $81 \%$ of the time in the -ATE set and only $51 \%$ of the time in the TA-E set.

The difference between the two sets for recall of the first item would be unimportant if it was simply a consequence of the -ATE stimuli being easier to recall. In fact, considering all six serial positions together, there was a main effect of type of stimulus, with the -ATE stimuli being recalled more often than the TA-E stimuli $[F(1,31)$ $\left.=26.14, M S_{\mathrm{e}}=203, p<.001\right]$. Therefore, it is worthwhile to consider a $2 \times 2$ ANOVA examining type of stimuli and Serial Position 1 versus Serial Position 2. This test indicated that the advantage for the -ATE stimuli over the TA-E stimuli was larger at Serial Position 1 than it was at Serial Position $2\left[F(1,31)=7.09, M S_{\mathrm{c}}=53.02\right.$, $p<.05]$.

There was little difference between the two types of stimuli for recall of the last item. In particular, recency, as measured by the difference between Serial Positions 5 and 6 , was almost identical, being $15 \%$ for the TA-E stimuli and $14 \%$ for the -ATE stimuli.

\section{Discussion}

Analysis 1 had shown that grouping primarily improves recall of the items adjoining the grouping. The interaction between type of stimuli and Serial Positions 3 and 4 in the present experiment suggests that grouping most improves recall of the phonemes adjoining the grouping. With the exception of the last serial position, the results of this experiment were predicted by the recovery explanation of grouping and hence serve as a confirmation of that explanation.

This experiment was not specifically designed to disconfirm any explanations of grouping. However, the present results are inconsistent with any explanation of boundary effects that assumes the first and last items of the group possess the critical property or cue. Instead, if there is a critical property or cue, it must be possessed by the first and last phonemes or the first and last sounds.

The overall better recall of the -ATE stimuli was unexpected, as the two sets of stimuli contained the same phonemes. There are several possible reasons for this better recall. First, although both sets contained four obvious words, the -ATE set also contained an uncommon word and a common name, and there was no attempt to control for word frequency. Thus, the better recall of the -ATE stimuli might have reflected lexical differences between the two sets. Second, although the two sets contained the same phonemes, they differed on the level of allophones, and hence they might have differed in overall degree of discriminability. (Allophones are smaller classes of sounds than phonemes that do not necessarily differ in ways that matter to a language.)

Third, the probability of recalling an item might depend upon whether the preceding item is recalled correctly. According to the recovery explanation, identifying one item defines the start of the subsequent item, making recovery of the subsequent item more likely. More mundanely, when a subject has difficulty recalling an item, the subject might deliberate on that item and create a delay that impairs recall of the subsequent items. In any case, the advantage for the -ATE stimuli on the first serial position might have carried over to Serial Positions 2 and 3 . This carryover would have been balanced by the advantage of the TA-E stimuli for Serial Position 3. However, the -ATE stimuli would have then acquired an advantage at Serial Position 4 which carried over to Serial Positions 5 and 6.

The only incorrect prediction of the recovery theory was for Serial Position 6, at which recency should have been larger for the TA-E set. Curiously, Darwin and Baddeley (1974) found this predicted effect: there was larger recency for the set $<A S H, A M, A G>$ than for the set $<$ SHA, MA, GA $>$.

In another study, I found larger recency for a set of items ending in ATE than for a set of items ending in AME (Frick, in press), suggesting that recency might be larger 
for items ending in stop consonants. In the present experiment, items in the -ATE set always ended in a stop consonant, whereas items in the TA-E set only sometimes ended in a stop consonant. When the next-to-last item ended in a stop consonant ( $D, K, P$, or $T)$, the recency effect in the TA-E set was 26\%; when the next-to-last item did not end in a stop consonant ( $F, L, M, V$, or $Z)$, the recency effect in the TA-E set was only $7 \%$.

Thus, the TA-E stimuli might have had an advantage for recency in that the critical phoneme was closer to the end of the presentation. However, this advantage might have been counterbalanced by the -ATE stimuli's advantage of having every item end in a stop consonant.

\section{SUMMARY AND CONCLUSION}

Rehearsal, chunking, and the limited directionality of transposition errors all predict that grouping should improve recall. In fact, each one of these factors might contribute (in one situation or another) to the improved recall produced by grouping. However, these factors cannot fully account for the effects of grouping. In fact, a combination of these factors cannot fully account for the effects of grouping, because none of these factors can account for the primacy and recency found for isolated transposition and substitution errors. There must be some additional source of the effects of grouping.

If the representation in ASTS is unparsed, and if the items represented in ASTS need to be identified prior to recall, items adjacent to boundaries would have an advantage in this process. This explanation accounts for the improved recall produced by grouping, and it explains why grouping primarily improves recall of the items adjoining the grouping. Because the representation in ASTS is hypothesized to be precategorical, this explanation accounts for why grouping most improves recall of the phonemes adjoining the grouping. Because grouping produces its effects during the process of recovery, nothing need happen during the presentation. Therefore, it is not surprising that grouping still has its effects when articulatory suppression is used or when there is no pause between groups.

These results also bear on various theories concerning the limited capacity of ASTS. First, the finding that rehearsal does not fully account for the effects of grouping poses difficulties for the trace-decay explanation of limited capacity. Second, the finding that chunking does not fully account for the effects of grouping poses difficulty for the theory that ASTS retains $7 \pm 2$ chunks. Finally, the boundary effects for isolated errors in recall are not predicted by Lee and Estes's $(1977,1981)$ model of shortterm memory, hence they would require a revision of that model. By providing support for the recovery explanation of grouping, the results of these experiments provide support for the hypothesis that ASTS retains an unparsed and precategorical representation, and that the capacity of ASTS is the number of items that can be represented with sufficient fidelity to be recovered.

\section{REFERENCES}

Adams, H. F. (1915). A note on the effect of rhythm on memory. Psychological Review, 22, 289-298.

BADDELEY, A. D. (1981). The concept of working memory: A view of its current state and probable future development. Cognition, 10, 17-23.

Baddeley, A. D. (1986). Working memory. Oxford: Clarendon Press.

BADDELEY, A. D., \& LEwIS, V. J. (1981). Inner active processes in reading: The inner voice, the inner ear and the inner eye. In $A$. $M$. Lesgold \& C. A. Perfetti (Eds.), Interactive processes in reading (pp. 107-129). Hillsdale, NJ: Erlbaum.

BADDELEY, A. D., LEWIS, V. J., \& VALLAR, G. (1984). Exploring the articulatory loop. Quarterly Joumal of Experimental Psychology, 36A, 233-252.

Baddeley, A. D., Thomson, N., \& Buchanan, M. (1975). Word length and the structure of short-term memory. Journal of Verbal Learning \& Verbal Behavior, 14, 575-589.

Besner, D., Davies, J., \& Daniels, S. (1981). Phonological processes in reading: The effects of concurrent articulation. Quarterly Joumal of Experimental Psychology, 33, 415-438.

Bower, G. H., \& Sprungston, F. (1970). Pauses as recoding points in letter series. Journal of Experimental Psychology, 83, 421-430.

CHI, M. T. H. (1976). Short-term memory limitations in children: Capacity or processing deficits? Memory \& Cognition, 4, 559-572.

ConRad, R. (1964). Acoustic confusions in immediate memory. British Journal of Psychology, 55, 75-84

ConRad, R. (1972). Speech and reading. In J. J. Kavanagh \& I. G. Mattingly (Eds.), Language by ear and by eye: The relationship between speech and reading (pp. 205-240). Cambridge MA: MIT Press.

Craik, F. I. M., \& Watkins, M. J. (1973). The role of rehearsal in short-term memory. Journal of Verbal Learning \& Verbal Behavior, 12, 599-607.

Crowder, R. G. , \& Morton, J. (1969). Precategorical acoustic storage (PAS). Perception \& Psychophysics, 5, 365-373.

Darwin, C. J., BadDeleY, A. D. (1974). Acoustic memory and the perception of speech. Cognitive Psychology, 6, 41-60.

ESTES, W. K. (1972). An associative basis of coding and organization in memory. In A. W. Melton \& E. Martin (Eds.), Coding processes in human memory (pp. 161-190). Washington DC: Winston.

Frick, R. W. (1985). Testing visual short-term memory: Simultaneous versus sequential presentations. Memory \& Cognition, 13, 346-356.

FrICK, R. W. (1988a). Issues of representation and limited capacity in the auditory short-term store. British Journal of Psychology, 79 213-240.

FRICK, R. W. (1988b). The role of memory in attenuations of the suffix effect. Memory \& Cognition, 16, 15-22.

FreK, R. W. (in press). Recency and the modality effect in immediate ordered recall. Canadian Journal of Psychology.

Gianutsos, R. (1972). Free recall of grouped words. Journal of Experimental Psychology, 95, 419-428.

Glassman, W. E. (1972). Subvocal activity and acoustic confusions in short-term memory. Journal of Experimental Psychology, 96 , 164-169.

Harcum, E. R. (1967). Parallel functions of serial learning and tachistoscopic pattern perception. Psychological Review, 74, 51-62.

HENDRIKX, A. J. P. (1984). Effects of grouping on recall latency. Acta Psychologica, 55, 1-18.

HinRICHS, J. V., \& Novick, L. R. (1982). Memory for numbers: Nominal vs, magnitude information. Memory \& Cognition, 10, 479-486.

Huttenlocher, J., \& BurKe, D. (1976). Why does memory span increase with age? Cognitive Psychology, 8, 1-31.

Kl.APP, S. T., Greim, D. M. \& Marshburn, E. A. (1981). Buffer storage of programmed articulation and articulatory loop: Two names for the same mechanism or two distinct components of short-term 
memory? In J. Long \& A. Baddeley (Eds.), Attention and performance (Vol. 9, pp. 459-472). Hillsdale, NJ: Erlbaum.

KlapP, S. T., Marshburn, E. A., \& Lester, P. T. (1983). Short-term memory does not involve the "working memory" of information processing: The demise of a common assumption. Journal of Experimental Psychology: General, 112, 240-264.

LeE, C. L., \& Estes, W. K. (1977). Order and position in primary memory for letter strings. Journal of Verbal Learning \& Verbal Behavior, 16, 395-418.

LEE, C. L., \& ESTES, W. K. (1981). Item and order information in shortterm memory: Evidence for multilevel perturbation processes. Journal of Experimental Psychology: Human Leaming \& Memory, 7, 149-169.

MiLler, G. A. (1956). The magical number seven, plus or minus two: Some limits on our capacity for processing information. Psychological Review, 63, 81-97.

Murdock, B. B., JR. (1974). Human memory: Theory and data. Potomac, MD: Erlbaum.

MuRRaY, D. J. (1967). The role of speech responses in short-term memory. Canadian Journal of Psychology, 21, 263-276.

MurRAY, D. J. (1968). Articulation and acoustic confusability in shortterm memory. Journal of Experimental Psychology, 78, 679-684.

Naveh-Benjamin, M., \& Jonides, J. (1984). Maintenance rehearsal: A two-component analysis. Joumal of Experimental Psychology: Learming, Memory, \& Cognition, 10, 369-385.

Peterson, L. R., \&ohnson, S. K. (1971). Some effect of minimizing articulation on short-term retention. Journal of Verbal Learning \& Verbal Behavior, 10, 346-354.

Reisberg, D., Smith, J. D., Baxter, D., \& Sonenshine, M. (in press). "Enacted" auditory images are ambiguous: "Pure" auditory images are not. Quarterly Joumal of Experimental Psychology.

Richardson, J. T. E., Greaves, D. E., \& SMith, M. M. C. (1980). Does articulatory suppression eliminate the phonemic similarity effect in short-term recall? Bulletin of the Psychonomic Society, 16, 417-420.

RYAN, J. (1969). Grouping and short-term memory: Different means and patterns of grouping. Quarterly Journal of Experimental Psychology, 21, 137-147.
Salame, P., \& Baddeley, A. D. (1982). Disruption of short-term memory by unattended speech: Implications for the structure of working memory. Journal of Verbal Learning \& Verbal Behavior, 21, 150-164.

SANDERS, A. F. (1975). Some remarks on short-term memory. In P. M. A. Rabbitt \& S. Dornic (Eds.), Attention and performance (Vol. 5, pp. 241-267). New York: Academic Press.

Sanders, A. F., \& Willemsen, E. M. (1978). The course of proactive interference in immediate probed recall. Acta Psychologica, 42, 133-144.

SANNOMIYA, M. (1985). The effect of suppressing subvocal speech on text processing during auditory and visual presentation. Japanese Psychological Research, 27, 41-44.

SCHWEICKERT, R., \& BoRUfF, B. (1986). Short-term memory capacity: Magic number or magic spell? Journal of Experimental Psychology: Leaming, Memory, \& Cognition, 12, 419-425.

Simon, H. A. (1974). How big is a chunk? Science, 183, 482-488.

STARR, A. S. (1923). The diagnostic value of the audito-vocal digit memory span. Psychological Clinic, 15, 61-84.

WiCKELGREN, W. (1964). Size of rehearsal group and short-term memory. Journal of Experimental Psychology, 68, 413-419.

WICKELGREN, W. (1967). rehearsal grouping and hierarchical organization of serial cues in short-term memory. Quarterly Journal of Experimental Psychology, 19, 97-102.

YNTema, D., Wozencraft, F., \& KLem, L. (1964, November). Immediate serial recall of digits presented at very high rates. Paper presented at the annual meeting of the Psychonomic Society, Niagara Falls, Ontario.

ZhaNG, G., \& Simon, H. A. (1985). STM capacity for Chinese words and idioms: Chunking and acoustical loop hypotheses. Memory \& Cognition, 13, 193-201.

(Manuscript received June 27, 1988; revision accepted for publication February 23, 1989.) 